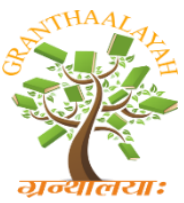

\author{
INTERNATIONAL JOURNAL OF RESEARCH - \\ GRANTHAALAYAH \\ A knowledge Repository
}

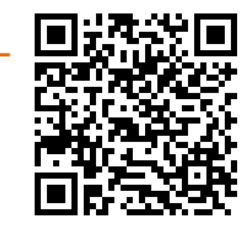

Science

\title{
EFFICACY STUDY OF PANCHARISHTA CHURNA IN DOOSHIVISHA WITH REFERENCE TO CONTACT DERMATITIS
}

\author{
Dr. Priya Popatrao Shinde ${ }^{* 1}$, Prof. Dr.V.P.Joglekar ${ }^{2}$ \\ *1 Assistant Professor, Agadtantra Department, S.S.N. Ayurved College Paikamal, Odisha, India \\ ${ }^{2}$ HOD \& Professor of Agadtantra Department, Tilak Ayurved Mahavidyalaya, Rasta Peth, Pune, \\ India
}

\begin{abstract}
The amazing journey of Ayurveda from its divine origin to the present day has left us wondering about enormous potential this science possesses. Its successful existence since time immemorial itself proves its scientific approach attributed to its unique principles that have remained unchanged till date. The Ayurveda is categorized into eight divisions, which collectively called Ashtang Ayurveda. Agadtantra is one among those branches.

This dissertation is an attempt to study the efficacy of "Pancharishta churna" in Dooshivish with special reference to contact dermatitis. The main purpose of this work is to help the person who is suffering from Contact dermatitis and wants to cure from the disease.

A Controlled Open prospective trial was carried out on 60 patients of 'Contact dermatitis', who were attended OPD \& having history of Dooshivish poisoning. 30 patients were treated by Pancharishta churna in study group and 30 patients were treated by Pancharishta churna \& Raktamokshana in control group at S.T.R.H. Pune.
\end{abstract}

Keywords: Pancharishta; Churna; Dooshivisha; Contact Dermatitis.

Cite This Article: Dr. Priya Popatrao Shinde, and Prof. Dr.V.P.Joglekar. (2017). "EFFICACY STUDY OF PANCHARISHTA CHURNA IN DOOSHIVISHA WITH REFERENCE TO CONTACT DERMATITIS." International Journal of Research - Granthaalayah, 5(10), 290-306. https://doi.org/10.29121/granthaalayah.v5.i10.2017.2305.

\section{Introduction}

Agadtantra deals with bites and poisoning of various animals like snakes, insects, spider, rat etc. and different type of compound poisons. [1] A definition of poison or toxin is difficult because a nontoxic substance may turn into toxin when administered in large dose or toxic substance can be used as medicine in very minimal quantities. But the Ayurveda give a nice definition of Visha.[2] Whatever substance after entering into the body creates panic in normal function of the basic tissues; destroy the wellbeing and threaten the life is called poison.[3] 
The present faulty food habits, polluted air, water, land and increased exposure to electromagnetic radiations, stressful lifestyle, unhealthy travelling habits are favorable condition to appear symptoms of dooshivisha. [4]

In today's era of urbanization and increasing demand of food for survival of human being more and more pesticides and fertilizers are used to yield more production. These synthetic fertilizers dramatically diminish nutrition value of food. So, we end up with food which are lack in viable nutrition and also loaded with residue form of other chemicals. Several medication, preservatives, colorings agent, additives are enter into our body in several way. Though they are in permitted range as per contemporary science but Ayurveda has different opinion regarding such toxins which are enter in our body in low quantity or low potency, they are termed as dooshivisha. Because Agadtantra explain that dooshivisha is a very specialized state of existence of weak poison in the body.

According to modern science dooshivisha can be consider as allergic condition of the body. Contact dermatitis or eczema is a polymorphic inflammation of the skin. It occurs at the site of contact with irritating or antigenic substances. In the acute phase there is occurrence of itching erythema, papules and vesicles, whereas in the chronic phase there is dryness, hyperkeratosis and sometimes fissures. Contact dermatitis is characterized by redness, itching, vesiculation and in more chronic form, scaly desquamation, resulting from exposure to environmental substances. ${ }^{[5]}$

Pancharishta churna is a combination of nimb, trikatu, triphala and haridra. In this combination, Nimb and haridra act as vishaghna, immune modulator and twakprasadak. Anulomana karma of triphala helps to excrete poison out of the body. Agnideepana karma of trikatu helps to modify digestive system. Thus Pancharishta churna act as vishaghna, anulomaka, agnideepaka and immune modulator. Due to these excellent properties of Pancharishta churna act as kushthghna and help to treat contact dermatitis effectively.

This dissertation is an attempt to study the efficacy of "Pancharishta churna" in Dooshivish with special reference to contact dermatitis. The main purpose of this work is to help the person who is suffering from Contact dermatitis and wants to cure from the disease.

\section{Need and Significance of the Study}

How the diseases are produced due incompatible factors (like Viruddhahara, asatmya ahara, ahita ahara, fast food, alcohol, antibiotics, steroids, medicines, pesticides, metals etc.) and Dooshivisha is the most important question \& it is yet to be answered.

This Clinical Study is an attempt to find out the solution for the contact dermatitis caused due to Dooshivish. It also finds the contact dermatitis caused by the adverse effect of some Modern medicines which have been used day by day to cure many diseases. In modern science medical treatment for contact dermatitis is not that much satisfactory due to recurrence and fewer options available. 


\subsection{Aim}

- "TO STUDY THE EFFICACY OF PANCHARISHTA CHURNA IN DOOSHIVISH WITH SPECIAL REFERENCE TO CONTACT DERMATITIS."

\subsection{Objectives}

- Collection of literary materials on Dooshivisha Concept.

- Collection of literary material on Kushtha.

- Collection of literary materials on Contact dermatitis.

- To study the standardization of ingredients as well as finished product (Panacharishta churna).

- To find out the action of Pancharishta churna in Dooshivish whether it breaks down the actual pathology or just gives symptomatic relief.

- To specify the role of Pancharishta churna in Dooshivishjanya contact dermatitis.

\section{Material and Method}

\section{MATRIAL: "Pancharishta churna" [6]}

Contains the following ingredients taken in equal quantity in the form of Churna.

Nimb: Twak, twaksar, seed, flower, leaves - 1 part.

Trikatu ---- 1 part

Haritaki ---- 1part

Bibhitaki ---- 1 part

Aamlaki ---- 1 part

Haridra ---- 1 part

Preparation of the Medicine

- Pancharishta churna is prepared by using standard textual method as per sharangdhar samhita.

- Raktamokshan will be done as per Sushruta samhita

\section{Method}

\subsection{Clinical Study}

- Source of the Data

Patients attending the O.P.D. of Seth Tarachand Hospital, Tilak Ayurved Mahavidyalaya, Rasta Peth,Pune with the complaints of contact dermatitis.

\section{- Clinical Trial}

Total 60 patients of contact dermatitis who have been attending OPD \& having history of DOOSHIVISH POISONING will be taken in to consideration. 
Among them 30 patients have been treated by MEDICATION in Group $A$ and rest 30 patients have been treated by MEDICATION+RAKTAMOKSHAN in Group B at S.T.R.H. Pune. These patients have been selected for the study irrespective of sex, religion, economical status, education, occupation etc.

\section{- Inclusion Criteria}

1) Patients having classical features of dooshivishajanya ${ }^{[7]}$ (contact dermatitis) have been taken as a subject to study.

2) These criteria have been employed before a desiring patient is included in this study.

3) Sex - Male / Female

4) Age - 16 to 70 years.

5) Patients having sign and symptoms like-

- Kandu

- Srava

- Pidaka

- Vedana

- Daha

- Twakvaivarnyata

- Exclusion criteria

1) Patients below age 16years and above 70 years

2) Patients associated with the major illnesses. HIV infection, hepatitis B infection and other blood disorder such as hemophilia are excluded from the study.

3) Pregnant woman.

4) Complication like deformities.

- Withdrawal criteria

$>$ Occurrence of serious adverse events.

$>$ Patient is not willing to continue the trial or to follow the assessment schedule.

$>$ Investigator feels that protocol has been violated or patient has become in-coperated.

$>$ Patients absent for follow up for 2days are considered as dropped out from this project.

- Mode of administration of the drug

The randomly selected patients were allocated into the following two groups and managed accordingly Study group \& Control group

A. Study group (Pancharishta churna)
A) Form
Churna
B) Dosage -
$1 \mathrm{gm}$ twice a days
C) Kala - after meal
D) Anupan -
lukewarm water
E) Route of Administration
Oral
F) Follow up -
7 th, 14 th, and days $28^{\text {th }}$

B. Control group (Pancharishta churna +Raktamokshan)

- Form- Abhayantara sevan of Pancharishta churna +Raktamokshan by application of leech.

- Dosage- 1 gm twice a day and Raktamokshan according to samyka lakshana.

- Kala-Pancharishta churna give after meal and Raktamokashan within interval of 7 days

- Anupan- lukewarm water for abhyantar sevan of churn 
- Route of Administration- Churna by Oral route and Raktamokshan by application of leech

- Follow up- 7th, 14th , and 28th day

\section{- Criteria for Assessment}

Clinical - Itching (kandu), erythema (vaivarnyata), vesicles (pidaka), oozing (srava),

Daha.

Laboratory - Blood investigation as per case proforma.

Scored score is used for assessment of clinical symptoms Using SCORAD

SCORAD = Scoring Atopic Dermatitis, clinical tool for assessing the severity of atopic dermatitis as objectively as possible

\section{Observations and Results}

Both subjective and objective parameters were considered for the assessment of the efficacy of Pancharishta churna in Dooshivisha with special reference to Contact dermatitis.

The responses obtained through clinical investigations were recorded and an honest effort was made to interpret the findings. A total of sixty patients participated in the clinical trial. The different data obtained from the study were:

Section A: Demographic data

Section B: Distribution of patients according to their personal history

Section C: Distribution of patient according to Disease

Section D: Data related to treatment response

\section{Section A: Demographic data:}

\begin{tabular}{|l|l|l|l|l|l|l|}
\multirow{3}{*}{ Age } & \multicolumn{2}{|c}{$\begin{array}{l}\text { Table 1: Distribution of the patients according to Age } \\
\text { Study }\end{array}$} & \multicolumn{2}{c|}{ Control } & \multicolumn{2}{c|}{ Total } \\
\cline { 2 - 7 } & count & \multicolumn{1}{|c|}{ Percentage } & count & percentage & Count & percentage \\
\hline $17-20$ & 04 & 13.33 & 5 & 16.67 & 9 & 15 \\
\hline $21-30$ & 5 & 16.67 & 5 & 16.67 & 10 & 16.67 \\
\hline $31-40$ & 11 & 36.67 & 10 & 33.33 & 21 & 35 \\
\hline $41-50$ & 6 & 20 & 4 & 13.33 & 10 & 16.67 \\
\hline $51-60$ & 2 & 6.67 & 2 & 6.67 & 4 & 6.67 \\
\hline $61-70$ & 1 & 3.33 & 2 & 6.67 & 3 & 5 \\
\hline
\end{tabular}

Table 2: Distribution of patients according to Gender

\begin{tabular}{|l|l|l|l|l|l|c|}
\hline \multirow{2}{*}{ Sex } & \multicolumn{2}{c|}{ Study } & \multicolumn{2}{c|}{ Control } & \multicolumn{2}{c|}{ Total } \\
\cline { 2 - 7 } & Count & Percentage & Count & Percentage & Count & Percentage \\
\hline Male & 20 & 66.67 & 21 & 70 & 41 & 68.33 \\
\hline Female & 10 & 33.33 & 9 & 30 & 19 & 31.67 \\
\hline
\end{tabular}


Table 3: Distribution of Patients according to Religion

\begin{tabular}{|l|l|l|l|l|l|c|}
\hline \multirow{2}{*}{ Religion } & \multicolumn{2}{|c|}{ Study } & \multicolumn{2}{c|}{ Control } & \multicolumn{2}{c|}{ Total } \\
\cline { 2 - 7 } & Count & \multicolumn{1}{c|}{ Percentage } & Count & Percentage & Count & Percentage \\
\hline Hindu & 28 & 93.3 & 26 & 86.7 & 54 & 90.00 \\
\hline Muslim & 02 & 6.7 & 04 & 13.3 & 06 & 10.00 \\
\hline
\end{tabular}

Table 4: Distribution of patients according to Domicile

\begin{tabular}{|l|l|l|l|l|l|c|}
\hline \multirow{2}{*}{ Domicile } & \multicolumn{2}{|c|}{ Study } & \multicolumn{2}{c|}{ Control } & \multicolumn{2}{c|}{ Total } \\
\cline { 2 - 7 } & Count & Percentage & Control & Percentage & Count & Percentage \\
\hline Urban & 25 & 83.33 & 21 & 70 & 46 & 76.67 \\
\hline Rural & 5 & 16.67 & 9 & 30 & 14 & 23.33 \\
\hline
\end{tabular}

Section B: Distribution of patients according to their personal history

Table 5: Distribution of patients according to Diet

\begin{tabular}{|c|c|c|c|c|c|c|}
\hline \multirow{2}{*}{$\begin{array}{l}\text { Personal } \\
\text { History } \\
\text { Vegetarian }\end{array}$} & \multicolumn{2}{|r|}{ Study } & \multicolumn{2}{|r|}{ Control } & \multicolumn{2}{|r|}{ Total } \\
\hline & $\begin{array}{l}\text { Count } \\
11\end{array}$ & $\begin{array}{l}\text { Percentage } \\
36.67\end{array}$ & ${ }_{9}^{\text {Count }}$ & ${ }_{30}$ Percentage & $\begin{array}{l}\text { Count } \\
20\end{array}$ & $\begin{array}{l}\text { Percentage } \\
33.33\end{array}$ \\
\hline Mixed & 19 & 63.33 & 21 & 70 & 40 & 66.67 \\
\hline
\end{tabular}

Table 6: Distribution of patients according to Occupation

\begin{tabular}{|l|l|l|l|l|l|l|}
\multirow{2}{*}{ Occupation } & \multicolumn{2}{|c|}{ Study } & \multicolumn{2}{c|}{ Control } & \multicolumn{2}{c|}{ Total } \\
\cline { 2 - 7 } & Count & Percentage & Count & Percentage & Count & Percentage \\
\hline Sedentory & 10 & 33.33 & 9 & 30 & 19 & 31.67 \\
\hline Moderate & 7 & 23.33 & 8 & 26.67 & 15 & 25 \\
\hline Heavy & 13 & 43.33 & 13 & 43.33 & 26 & 43.33 \\
\hline
\end{tabular}

Table 7: Distribution of patients according to Economical status

\begin{tabular}{|c|c|c|c|c|c|c|}
\hline \multirow[b]{2}{*}{$\begin{array}{l}\text { Religion } \\
\text { Poor }\end{array}$} & \multicolumn{2}{|r|}{ Study } & \multicolumn{2}{|r|}{ Control } & \multicolumn{2}{|r|}{ Total } \\
\hline & ${ }_{8}^{\text {Count }}$ & $\begin{array}{l}\text { Percentage } \\
26.67\end{array}$ & $6^{\text {Count }}$ & $20^{\text {Percentage }}$ & $\begin{array}{l}\text { Count } \\
14\end{array}$ & $\begin{array}{l}\text { Percentage } \\
23.33\end{array}$ \\
\hline Middle & 21 & 70 & 13 & 43.33 & 34 & 56.67 \\
\hline High & 1 & 3.33 & 1 & 3.33 & 2 & 3.33 \\
\hline
\end{tabular}

Table 8: Distribution of patients according to Bowel movements

\begin{tabular}{|c|c|c|c|c|c|c|}
\hline Bowel & & Study & & Control & & Total \\
\hline $\begin{array}{l}\text { movements } \\
\text { Regular }\end{array}$ & $\begin{array}{l}\text { Count } \\
13\end{array}$ & $\begin{array}{l}\text { Percentage } \\
43.33\end{array}$ & $9_{9}^{\text {Count }}$ & ${ }_{30}^{\text {Percentage }}$ & $\begin{array}{l}\text { Count } \\
22\end{array}$ & $\begin{array}{l}\text { Percentage } \\
36.67\end{array}$ \\
\hline Constipated & 17 & 56.67 & 21 & 70 & 38 & 63.33 \\
\hline
\end{tabular}

Table 9: Distribution of patients according to Prakruti

\begin{tabular}{|l|l|l|l|l|l|l|}
\hline \multirow{2}{*}{ Prakruti } & \multicolumn{2}{|c|}{ Study } & \multicolumn{2}{c|}{ Control } & \multicolumn{2}{c|}{ Total } \\
\cline { 2 - 7 } & Count & Percentage & Count & Percentage & Count & Percentage \\
\hline Vatapitta & 6 & 20 & 8 & 26.67 & 14 & 23.33 \\
\hline Vatakapha & 15 & 50 & 14 & 46.67 & 29 & 48.33 \\
\hline KaphaPitta & 9 & 30 & 8 & 26.67 & 17 & 28.33 \\
\hline
\end{tabular}


Table 10: Distribution of patients according to Mental Stress

\begin{tabular}{|l|l|l|l|l|l|c|}
\hline \multirow{2}{*}{ Mental Stress } & \multicolumn{2}{|c|}{ Study } & \multicolumn{2}{c|}{ Control } & \multicolumn{2}{c|}{ Total } \\
\cline { 2 - 7 } & Count & Percentage & Count & Percentage & Count & Percentage \\
\hline Present & 21 & 70 & 19 & 63.33 & 40 & 66.67 \\
\hline Absent & 9 & 30 & 11 & 36.67 & 20 & 33.33 \\
\hline
\end{tabular}

Table 11: Distribution of patients according to Addictions

\begin{tabular}{|l|l|l|l|l|l|l|}
\hline \multirow{2}{*}{\multicolumn{1}{|c|}{ Addictions }} & \multicolumn{2}{c|}{ Study } & \multicolumn{2}{c|}{ Control } & \multicolumn{2}{c|}{ Total } \\
\cline { 2 - 7 } & Count & \% & Count & \multicolumn{1}{c|}{ Count } & Percentage \\
\hline No addiction & 4 & 13.33 & 5 & 16.67 & 9 & 15 \\
\hline Tea/Coffee & 10 & 33.33 & 9 & 30 & 19 & 31.67 \\
\hline Alcohol & 8 & 26.67 & 7 & 23.33 & 15 & 25 \\
\hline Smoking & 3 & 10 & 4 & 13.33 & 7 & 11.67 \\
\hline Smoking + Alcohol & 3 & 10 & 4 & 13.33 & 7 & 11.67 \\
\hline Other & 2 & 6.67 & 1 & 3.33 & 3 & 5 \\
\hline
\end{tabular}

Section C - Distribution of patient according to Disease

Table 12: Distribution of patients according to Duration of disease

\begin{tabular}{|l|l|l|l|l|l|l|}
\hline \multirow{2}{*}{$\begin{array}{c}\text { Duration of } \\
\text { disease }\end{array}$} & \multicolumn{2}{|c|}{ Study } & \multicolumn{2}{c|}{ Control } & \multicolumn{2}{c|}{ Total } \\
\cline { 2 - 7 } & Count & Percentage & Count & Percentage & Count & Percentage \\
\hline Up to 1 year & 18 & 60 & 14 & 46.66667 & 32 & 53.33333 \\
\hline Above 1 year & 12 & 40 & 16 & 53.33333 & 18 & 30 \\
\hline
\end{tabular}

Table 13: Distribution of patients according to H/o Dooshivisha

\begin{tabular}{|l|l|l|l|l|l|l|l|}
\hline \multicolumn{1}{|c|}{ History of Dooshivisha } & \multicolumn{2}{c|}{ Study } & \multicolumn{2}{c|}{ Control } & \multicolumn{2}{c|}{ Total } \\
& Count & $\boldsymbol{\%}$ & Count & \% & Count & \multicolumn{1}{c|}{$\%$} \\
History Of Jangama visha & 3 & 10 & 1 & 3.33 & 4 & 6.67 \\
History Of Sthavara visha & 2 & 6.67 & 1 & 3.33 & 3 & 5 \\
\hline History Of Viruddhahara & 13 & 43.33 & 19 & 63.33 & 32 & 53.33 \\
\hline History Of Allopathic drugs & 10 & 33.33 & 9 & 30 & 19 & 31.67 \\
\hline
\end{tabular}

Table 14: Distribution of patients according to Aggravating factors

\begin{tabular}{|c|c|c|c|c|c|c|}
\hline Aggravating & & ly & & ntrol & & \\
\hline Factors & Count & $\%$ & Count & $\%$ & Count & $\%$ \\
\hline Cold Season & 4 & 13.33 & 3 & & 7 & 11.67 \\
\hline Non-veg food & 10 & 33.33 & 13 & 43.33 & 23 & 38.33 \\
\hline Sun exposure & 3 & 10 & 4 & 13.33 & 7 & 11.67 \\
\hline Sweating & 5 & 16.67 & 3 & 10 & 8 & 13.33 \\
\hline Occupation & 8 & 26.67 & 7 & 23.33 & 15 & 25 \\
\hline
\end{tabular}

Section D - Data related to treatment response

T-Test: Two-Sample Assuming Equal Variances 
Table 15: Effectiveness of treatment on SCORAD Score difference between two groups

\begin{tabular}{|l|l|l|l|l|}
\hline \multicolumn{1}{|c|}{ Group } & \multicolumn{1}{|c|}{ Mean of SCORAD diff. } & Variance & Observations & $\begin{array}{c}\text { P value } \\
\mathbf{P}(\mathbf{T}<=\mathbf{t}) \text { two-tail }\end{array}$ \\
\hline Study & 25.13 & 37.15 & 30 & $\mathbf{0 . 2 1 1 4 2 1 3 2 1}$ \\
Control & 27.23 & 45.70 & 30 & \\
\hline
\end{tabular}

Table 16: Effectiveness on SCORAD Score between two groups according to Age

\begin{tabular}{|c|c|c|c|c|c|c|c|}
\hline \multirow[b]{2}{*}{ Age } & \multicolumn{2}{|c|}{ Mean of SCORAD diff } & \multicolumn{2}{|c|}{ Variance } & \multicolumn{2}{|c|}{ Observations } & \multirow{2}{*}{$\begin{array}{c}P \text { value } \\
\mathbf{P}(\mathrm{T}<=\mathrm{t}) \text { two- } \\
\text { tail }\end{array}$} \\
\hline & Study & Control & Study & Control & Study & Control & \\
\hline $17-20$ & 39 & 29.2 & 102 & 31.7 & 4 & 5 & 0.105534414 \\
\hline $21-30$ & 20 & 24.2 & 22 & 27.2 & 5 & 5 & 0.21739574 \\
\hline $31-40$ & 28.67 & 28.83 & 35.15 & 43.79 & 12 & 12 & 0.948775274 \\
\hline $41-50$ & 25.17 & 20.25 & 23.37 & 58.92 & 6 & 4 & 0.244090401 \\
\hline $51-60$ & 26.5 & 35 & 0.5 & 18 & 2 & 2 & 0.107731402 \\
\hline $61-70$ & 25 & 26.5 & \#DIV/0! & 0.5 & 1 & 2 & $\mathbf{0 . 3 3 3 3 3 3 3 3 3}$ \\
\hline
\end{tabular}

Table 17: Effectiveness on SCORAD Score between two groups according to Sex

\begin{tabular}{|c|c|c|c|c|c|c|c|}
\hline \multirow[b]{2}{*}{ Sex } & \multicolumn{2}{|c|}{ Mean of SCORAD diff. } & \multicolumn{2}{|c|}{ Variance } & \multicolumn{2}{|c|}{ Observations } & \multirow{2}{*}{$\begin{array}{c}\text { P value } \\
P(T<=t) \text { two-tail }\end{array}$} \\
\hline & Study & Control & Study & Control & Study & Control & \\
\hline Male & 25.3 & 27.57 & 44.85 & 45.46 & 20 & 21 & 0.285990571 \\
\hline Female & 24.8 & 26.44 & 24.84 & 51.03 & 10 & 9 & 0.564872105 \\
\hline
\end{tabular}

Table 18: Effectiveness on SCORAD Score between two groups according to Religion

\begin{tabular}{|l|l|l|l|l|l|l|l|}
\hline \multirow{2}{*}{ Religion } & \multicolumn{2}{|c|}{$\begin{array}{c}\text { Mean of SCORAD } \\
\text { diff. }\end{array}$} & \multicolumn{2}{c|}{ Variance } & \multicolumn{2}{c|}{ Observations } & \multirow{2}{*}{$\begin{array}{c}\text { P value } \\
\text { P(T<=t) two-tail }\end{array}$} \\
\cline { 2 - 7 } & Study & Control & Study & Control & Study & Control & \\
\hline Hindu & 25.43 & 27.27 & 37.37 & 50.36 & 28 & 26 & $\mathbf{0 . 3 1 0 8 7 8 8 2 7}$ \\
\hline Muslim & 21 & 27 & 32 & 22 & 2 & 4 & $\mathbf{0 . 2 3 4 1 8 1 3 3 6}$ \\
\hline
\end{tabular}

Table 19: Effectiveness on SCORAD Score between two groups according to Domicile

\begin{tabular}{|l|l|l|l|l|l|l|l|}
\multirow{2}{*}{ Domicile } & \multicolumn{2}{|c|}{$\begin{array}{c}\text { Mean of SCORAD } \\
\text { diff. }\end{array}$} & \multicolumn{2}{c|}{ Variance } & \multicolumn{2}{c|}{ Observations } & \multirow{2}{*}{$\begin{array}{c}\text { P value } \\
\text { P(T<=t) two-tail }\end{array}$} \\
\cline { 2 - 7 } & Study & Control & Study & Control & Study & Control & \\
\hline Urban & 20.4 & 30.22 & 24.8 & 29.19 & 5 & 9 & $\mathbf{0 . 0 0 5 8 4 3 5 3 4}$ \\
\hline Rural & 26.08 & 25.95 & 35.16 & 48.85 & 25 & 21 & $\mathbf{0 . 9 4 6 8 6 8 4 3 1}$ \\
\hline
\end{tabular}

Table 20: Effectiveness on SCORAD Score between two groups according to Occupation

\begin{tabular}{|l|l|l|l|l|l|l|l|}
\hline \multirow{2}{*}{ Occupation } & \multicolumn{2}{|c|}{$\begin{array}{c}\text { Mean of SCORAD } \\
\text { diff. }\end{array}$} & \multicolumn{2}{c|}{ Variance } & \multicolumn{2}{c|}{ Observations } & \multirow{2}{*}{$\begin{array}{c}\text { P value } \\
\text { P(T<=t) two-tail }\end{array}$} \\
\cline { 2 - 7 } & Study & Control & Study & Control & Study & Control & \\
\hline Sedentory & 21.7 & 28.22 & 37.34 & 22.44 & 10 & 9 & $\mathbf{0 . 0 1 9 5 7 2 2 2 9}$ \\
\hline Moderate & 26.57 & 26.87 & 54.29 & 71.55 & 7 & 8 & $\mathbf{0 . 9 4 2 4 8 1 0 5 3}$ \\
\hline Heavy & 27 & 26.77 & 19.833 & 52.70 & 13 & 13 & $\mathbf{0 . 9 2 2 9 8 0 2 5 3}$ \\
\hline
\end{tabular}


Table 21: Effectiveness on SCORAD Score between two groups according to Income

\begin{tabular}{|c|c|c|c|c|c|c|c|}
\hline \multirow[b]{2}{*}{ Income } & \multicolumn{2}{|c|}{$\begin{array}{l}\text { Mean of SCORAD } \\
\text { diff. }\end{array}$} & \multicolumn{2}{|c|}{ Variance } & \multicolumn{2}{|c|}{ Observations } & \multirow{2}{*}{$\begin{array}{c}\text { P value } \\
\mathbf{P}(\mathrm{T}<=\mathrm{t}) \mathrm{two-} \\
\text { tail }\end{array}$} \\
\hline & Study & Control & Study & Control & Study & Control & \\
\hline Poor & 29.87 & 22.17 & 26.70 & 38.57 & 8 & 6 & 0.026064314 \\
\hline Middle & 23.71 & 28.30 & 30.11 & 41.77 & 21 & 23 & 0.015357937 \\
\hline High & \multicolumn{7}{|c|}{ Not comparable } \\
\hline
\end{tabular}

Table 22: Effectiveness on SCORAD Score between two groups according to Diet

\begin{tabular}{|c|c|c|c|c|c|c|c|}
\hline \multirow[t]{2}{*}{ Diet } & \multicolumn{2}{|c|}{$\begin{array}{c}\text { Mean of SCORAD } \\
\text { diff. }\end{array}$} & \multicolumn{2}{|c|}{ Variance } & \multicolumn{2}{|c|}{ Observations } & \multirow{2}{*}{$\begin{array}{c}P \text { value } \\
\mathbf{P}(T<=t) \text { two- } \\
\text { tail }\end{array}$} \\
\hline & Study & Control & Study & Control & Study & Control & \\
\hline Vegetarian & 24.54 & 26.44 & 27.47 & 62.28 & 11 & 9 & 0.527226847 \\
\hline Mixed & 25.47 & 26.94 & 44.26 & 43.35 & 19 & 18 & 0.503801708 \\
\hline
\end{tabular}

Table 23: Effectiveness on SCORAD Score between two groups according to Mental stress

\begin{tabular}{|c|c|c|c|c|c|c|c|}
\hline \multirow[t]{2}{*}{$\begin{array}{c}\text { Mental } \\
\text { stress }\end{array}$} & \multicolumn{2}{|c|}{$\begin{array}{c}\text { Mean of SCORAD } \\
\text { diff. }\end{array}$} & \multicolumn{2}{|c|}{ Variance } & \multicolumn{2}{|c|}{ Observations } & \multirow{2}{*}{$\begin{array}{c}\text { P value } \\
\mathbf{P}(\mathbf{T}<=\mathbf{t}) \text { two- } \\
\text { tail }\end{array}$} \\
\hline & Study & Control & Study & Contrl & Study & Control & \\
\hline $\begin{array}{l}\text { Present } \\
\text { Absent }\end{array}$ & $\begin{array}{l}25.19 \\
25\end{array}$ & $\begin{array}{l}26.85 \\
28\end{array}$ & $\begin{array}{l}24.26 \\
74\end{array}$ & $\begin{array}{l}50.13 \\
40.44\end{array}$ & $\begin{array}{l}21 \\
9\end{array}$ & $\begin{array}{l}20 \\
10\end{array}$ & $\begin{array}{l}0.38704677 \\
0.39605499\end{array}$ \\
\hline
\end{tabular}

Table 24: Effectiveness on SCORAD Score between two groups according to Prakruti

\begin{tabular}{|c|c|c|c|c|c|c|c|}
\hline \multirow[t]{2}{*}{ Prakruti } & \multicolumn{2}{|c|}{$\begin{array}{c}\text { Mean of SCORAD } \\
\text { diff. }\end{array}$} & \multicolumn{2}{|c|}{ Variance } & \multicolumn{2}{|c|}{ Observations } & \multirow{2}{*}{$\begin{array}{c}\text { P value } \\
P(T<=t) \text { two- } \\
\text { tail }\end{array}$} \\
\hline & Study & Control & Study & Control & Study & Control & \\
\hline $\begin{array}{l}\text { VataKapha } \\
\text { VataPitta }\end{array}$ & $\begin{array}{l}23.5 \\
27\end{array}$ & $\begin{array}{l}25.75 \\
26.58\end{array}$ & $\begin{array}{l}15.9 \\
40.57\end{array}$ & $\begin{array}{l}77.36 \\
46.88\end{array}$ & $\begin{array}{l}6 \\
15 \\
\end{array}$ & $\begin{array}{l}8 \\
14\end{array}$ & $\begin{array}{l}0.573205483 \\
0.862664006 \\
\end{array}$ \\
\hline KaphaPitta & 23.11 & 29.87 & 40.61 & 13.55 & 9 & 8 & 0.018880974 \\
\hline
\end{tabular}

Table 25: Effectiveness on SCORAD Score between two groups according to satva

\begin{tabular}{|c|c|c|c|c|c|c|c|}
\hline \multirow{4}{*}{$\begin{array}{l}\text { Bowel } \\
\text { movements } \\
\text { Madya } \\
\text { Avar }\end{array}$} & \multicolumn{2}{|c|}{$\begin{array}{c}\text { Mean of } \\
\text { SCORAD diff. }\end{array}$} & \multicolumn{2}{|c|}{ Variance } & \multicolumn{2}{|c|}{ Observations } & \multirow{4}{*}{$\begin{array}{c}\text { P value } \\
\mathbf{P}(\mathbf{T}<=t) \text { two- } \\
\text { tail } \\
0.699844138 \\
0.30518002\end{array}$} \\
\hline & Study & Control & Study & Control & Study & Control & \\
\hline & 26.81 & 27.57 & 20.16 & 45.46 & 16 & 21 & \\
\hline & 23.22 & 26.44 & 52.18 & 51.03 & 14 & 9 & \\
\hline
\end{tabular}

Table 26: Effectiveness on SCORAD Score between two groups according to Bowel movements

\begin{tabular}{|c|c|c|c|c|c|c|c|}
\hline \multirow[t]{2}{*}{$\begin{array}{c}\text { Bowel } \\
\text { movements }\end{array}$} & \multicolumn{2}{|c|}{$\begin{array}{c}\text { Mean of } \\
\text { SCORAD diff. }\end{array}$} & \multicolumn{2}{|c|}{ Variance } & \multicolumn{2}{|c|}{ Observations } & \multirow{2}{*}{$\begin{array}{c}P \text { value } \\
\mathbf{P}(\mathbf{T}<=t) \text { two- } \\
\text { tail }\end{array}$} \\
\hline & Study & Control & Study & Control & Study & Control & \\
\hline Regular & 25.81 & 27.44 & 65.76 & 66.78 & 11 & 9 & 0.66187379 \\
\hline Constipated & 24.73 & 27.14 & 22.87 & 39.53 & 19 & 21 & 0.184692312 \\
\hline
\end{tabular}


Table 27: Effectiveness on SCORAD Score between two groups according to Addiction

\begin{tabular}{|c|c|c|c|c|c|c|c|}
\hline \multirow[b]{2}{*}{ Addiction } & \multicolumn{2}{|c|}{$\begin{array}{c}\text { Mean of } \\
\text { SCORAD diff. }\end{array}$} & \multicolumn{2}{|c|}{ Variance } & \multicolumn{2}{|c|}{ Observations } & \multirow[t]{2}{*}{$\begin{array}{c}\text { P value } \\
P(T<=t) \text { two-tail }\end{array}$} \\
\hline & Study & Control & Study & Control & Study & Control & \\
\hline No addiction & 12 & 27.5 & \#DIV/0! & 84.5 & 1 & 2 & 0.39991722 \\
\hline Tea/coffee & 27.2 & 25.9 & 57.51 & 60.32 & 10 & 10 & 0.70933152 \\
\hline Alcohol & 24.87 & 27.71 & 16.41 & 58.90 & 8 & 7 & 0.37733706 \\
\hline Smoking & 22.67 & 23.5 & 0.33 & 27.67 & 3 & 4 & 0.8003293 \\
\hline $\begin{array}{l}\text { Smoking } \\
\text { Alcohol }\end{array}$ & 27 & 31.4 & 17.2 & 18.8 & 6 & 5 & 0.12011978 \\
\hline Other & 15 & 29 & \#DIV/0! & 18 & 1 & 2 & 0.22625107 \\
\hline
\end{tabular}

Table 28: Effectiveness on SCORAD Score between two groups according to Aggravating

\begin{tabular}{|c|c|c|c|c|c|c|c|}
\hline \multirow{3}{*}{$\begin{array}{l}\text { Aggravating } \\
\text { Factor }\end{array}$} & & & \multicolumn{2}{|c|}{ Factor } & \multirow{2}{*}{\multicolumn{2}{|c|}{ Observations }} & \multirow{3}{*}{$\begin{array}{c}\text { P value } \\
\mathbf{P}(\mathrm{T}<=\mathrm{t}) \mathrm{two}- \\
\text { tail }\end{array}$} \\
\hline & \multicolumn{2}{|c|}{$\begin{array}{c}\text { Mean of SCORAD } \\
\text { diff. }\end{array}$} & \multicolumn{2}{|c|}{ Variance } & & & \\
\hline & Study & Control & Study & Control & Study & Control & \\
\hline Cold Season & 30.5 & 30.33 & 55 & 32.33 & 4 & 3 & 0.97556006 \\
\hline Non-veg food & 25 & 26.67 & 20.67 & 48.56 & 10 & 13 & 0.51331007 \\
\hline Sun exposure & 21 & 30 & 16.67 & 16.67 & 4 & 4 & 0.02064552 \\
\hline Sweating & 25.4 & 27 & 67.8 & 13 & 5 & 3 & 0.7661086 \\
\hline Occupation & 24.43 & 25.43 & 36.28 & 85.952 & 7 & 7 & 0.81491005 \\
\hline
\end{tabular}

Table 29: Effectiveness on Kandu between two groups

\begin{tabular}{|c|c|c|c|c|c|c|c|c|c|}
\hline \multirow[b]{2}{*}{ Group } & \multicolumn{3}{|c|}{ BT } & \multicolumn{3}{|c|}{ AT } & \multirow{2}{*}{$\begin{array}{l}\text { Mean } \\
\text { Diff. }\end{array}$} & \multirow{2}{*}{ Paired 't' } & \multirow[b]{2}{*}{$\mathbf{P}$} \\
\hline & $\mathbf{N}$ & Mean & SD & $\mathbf{N}$ & Mean & SD & & & \\
\hline Study & 30 & 2.33 & 0.55 & 30 & 0.6 & 0.56 & 1.73 & 18.23 & $\mathrm{p}<0.001$ \\
\hline Control & 30 & 2.3 & 0.59 & 30 & 0.43 & 0.50 & 1.86 & 23.55 & $p<0.001$ \\
\hline
\end{tabular}

The mean scores of Kandu before treatment were 2.33 and 2.3 for the study and control groups respectively. After treatment, it decreased to 0.60 and 0.43 for study and control group respectively. The mean difference noticed was 1.73 and 1.86 respectively. The paired' $t$ ' value 18.22 and 23.54 respectively, shows that this mean difference is very highly significant at $\mathrm{p}<0.001$ for study and control group.

Table 30: Effectiveness on Vedana between two groups

\begin{tabular}{|c|c|c|c|c|c|c|c|c|c|}
\hline \multirow{2}{*}{ Group } & \multicolumn{3}{|c|}{ BT } & \multicolumn{3}{|c|}{ AT } & \multirow{2}{*}{$\begin{array}{c}\text { Mean } \\
\text { Diff. }\end{array}$} & \multirow{2}{*}{ Paired ' $t$ ' } & \multirow{2}{*}{$\mathbf{P}$} \\
\hline & $\mathbf{N}$ & Mean & SD & $\mathbf{N}$ & Mean & SD & & & \\
\hline Study & 30 & 1.8 & 1.8 & 30 & 0.43 & 0.50 & 1.37 & 13.47 & $\mathrm{p}<0.001$ \\
\hline Control & 30 & 1.43 & 0.50 & 30 & 0.3 & 0.47 & 1.13 & 17.95 & $p<0.001$ \\
\hline
\end{tabular}

The mean scores of Vedana before treatment were 1.88 and 1.43 for the study and control groups respectively. After treatment, it decreased to 0.43 and 0.3 for study and control group respectively. The mean difference noticed was 1.37 and 1.13 respectively. The paired' $t$ ' value 13.47 and 17.95 respectively, shows that this mean difference is very highly significant at $\mathrm{p}<0.001$ for study and control group. 
Table 31: Effectiveness on Shoth between two groups

\begin{tabular}{|c|c|c|c|c|c|c|c|c|c|}
\hline \multirow{2}{*}{ Group } & \multicolumn{3}{|c|}{ BT } & \multicolumn{3}{|c|}{ AT } & \multirow{2}{*}{$\begin{array}{c}\text { Mean } \\
\text { Diff. }\end{array}$} & \multirow{2}{*}{ Paired $^{6} t$ ' } & \multirow{2}{*}{$\mathbf{P}$} \\
\hline & $\mathbf{N}$ & Mean & SD & $\mathbf{N}$ & Mean & SD & & & \\
\hline Study & 30 & 1.77 & 0.63 & 30 & 0.5 & 0.57 & 1.27 & 15.42 & $\mathrm{p}<0.001$ \\
\hline Control & 30 & 1.77 & 0.50 & 30 & 0.4 & 0.50 & 1.37 & 15.27 & $p<0.001$ \\
\hline
\end{tabular}

The mean scores of Shoth before treatment were 1.77 and 1.77 for the study and control groups respectively. After treatment, it decreased to 0.5 and 0.4 for study and control group respectively. The mean difference noticed was 1.27 and 1.37 respectively. The paired ' $t$ ' value 15.42 and 15.27 respectively, shows that this mean difference is very highly significant at $\mathrm{p}<0.001$ for study and control group.

Table 32: Effectiveness on Pidaka between two groups

\begin{tabular}{|c|c|c|c|c|c|c|c|c|c|}
\hline \multirow{2}{*}{ Group } & \multicolumn{3}{|c|}{ BT } & \multicolumn{3}{|c|}{ AT } & \multirow{2}{*}{$\begin{array}{c}\text { Mean } \\
\text { Diff. }\end{array}$} & \multirow{2}{*}{ Paired ' $\mathbf{t}$ ' } & \multirow{2}{*}{$\mathbf{P}$} \\
\hline & $\mathbf{N}$ & Mean & SD & $\mathbf{N}$ & Mean & SD & & & \\
\hline Study & 30 & 1.8 & 0.76 & 30 & 0.57 & 0.50 & 1.23 & 13.40 & $\mathrm{p}<0.001$ \\
\hline Control & 30 & 1.43 & 0.63 & 30 & 0.33 & 0.48 & 1.1 & 12.53 & $\mathrm{p}<0.001$ \\
\hline
\end{tabular}

The mean scores of Pidaka before treatment were 1.8 and 1.43 for the study and control groups respectively. After treatment, it decreased to 0.57 and 0.33 for study and control group respectively. The mean difference noticed was 1.23 and 1.1 respectively. The paired' $t$ ' value 13.40 and 12.53 respectively shows that this mean difference is significant at $p<0.001$ for study and control group.

Table 33: Effectiveness on Srava between two groups

\begin{tabular}{|c|c|c|c|c|c|c|c|c|c|}
\hline \multirow[b]{2}{*}{ Group } & \multicolumn{3}{|c|}{ BT } & \multicolumn{3}{|c|}{ AT } & \multirow{2}{*}{$\begin{array}{l}\text { Mean } \\
\text { Diff. }\end{array}$} & \multirow{2}{*}{ Paired $^{`} \mathbf{t}^{\prime}$} & \multirow{2}{*}{$\mathbf{P}$} \\
\hline & $\mathbf{N}$ & Mean & SD & $\mathbf{N}$ & Mean & SD & & & \\
\hline Study & 30 & 1.8 & 0.89 & 30 & 0.57 & 0.63 & 1.23 & 11.89 & $\mathrm{p}<0.001$ \\
\hline Control & 30 & 1.53 & 0.50 & 30 & 0.4 & 0.50 & 1.13 & 12.23 & $\mathrm{p}<0.001$ \\
\hline
\end{tabular}

The mean scores of Srava before treatment were 1.8 and 1.53 for the study and control groups respectively. After treatment, it decreased to 0.57 and 0.4 for study and control group respectively. The mean difference noticed was 1.23 and 1.13 respectively. The paired' $t$ ' value 11.89 and 12.23 respectively, shows that this mean difference is significant at $p<0.001$ for both study and control group.

Table 34: Effectiveness on Daha between two groups

\begin{tabular}{|c|c|c|c|c|c|c|c|c|c|}
\hline \multirow{2}{*}{ Group } & \multicolumn{3}{|c|}{ BT } & \multicolumn{3}{|c|}{ AT } & \multirow{2}{*}{$\begin{array}{c}\text { Mean } \\
\text { Diff. }\end{array}$} & \multirow{2}{*}{ Paired ' $t$ ' } & \multirow{2}{*}{$\mathbf{P}$} \\
\hline & $\mathbf{N}$ & Mean & SD & $\mathbf{N}$ & Mean & SD & & & \\
\hline Study & 30 & 1.7 & 0.70 & 30 & 0.53 & 0.51 & 1.17 & 16.86 & $\mathrm{p}<0.001$ \\
\hline Control & 30 & 1.27 & 0.52 & 30 & 0.27 & 0.45 & 1 & 20.86 & $\mathrm{p}<0.001$ \\
\hline
\end{tabular}

The mean scores of Daha before treatment were 1.7 and 1.27 for the study and control groups respectively. After treatment, it decreased to 0.53 and 0.27 for study and control group respectively. The mean difference noticed was 1.17 and 1.0 respectively. The paired' $t$ ' value 
16.86 and 20.86 respectively shows that this mean difference is significant at $\mathrm{p}<0.001$ for both study and control group.

Table 35: Effectiveness on Twakvaivarnya between two groups

\begin{tabular}{|c|c|c|c|c|c|c|c|c|c|}
\hline \multirow{2}{*}{ Group } & \multicolumn{3}{|c|}{ BT } & \multicolumn{3}{|c|}{ AT } & \multirow{2}{*}{$\begin{array}{c}\text { Mean } \\
\text { Diff. }\end{array}$} & \multirow{2}{*}{ Paired't' } & \multirow{2}{*}{$\mathbf{P}$} \\
\hline & $\mathbf{N}$ & Mean & SD & $\mathbf{N}$ & Mean & SD & & & \\
\hline Study & 30 & 2.1 & 0.76 & 30 & 0.8 & 0.61 & 1.3 & 15.28 & $\mathrm{p}<0.001$ \\
\hline Control & 30 & 1.83 & 0.70 & 30 & 0.63 & 0.49 & 1.2 & 16.15 & $p<0.001$ \\
\hline
\end{tabular}

The mean scores of Twakvaivarnya before treatment were 2.1 and 1.83 for the study and control groups respectively. After treatment, it decreased to 0.8 and 0.63 for study and control group respectively. The mean difference noticed was 1.3 and 1.2 respectively. The paired ' $t$ ' value 15.28 and 16.15 respectively shows that this mean difference is significant at $\mathrm{p}<0.001$ for both study and control group.

Table 36: Effectiveness on Twakjadyata between two groups

\begin{tabular}{|c|c|c|c|c|c|c|c|c|c|}
\hline \multirow{2}{*}{ Group } & \multicolumn{3}{|c|}{ BT } & \multicolumn{3}{|c|}{ AT } & \multirow{2}{*}{$\begin{array}{c}\text { Mean } \\
\text { Diff. }\end{array}$} & \multirow{2}{*}{ Paired ' $t$ ' } & \multirow{2}{*}{$\mathbf{P}$} \\
\hline & $\mathbf{N}$ & Mean & SD & $\mathbf{N}$ & Mean & SD & & & \\
\hline Study & 30 & 2.13 & 0.68 & 30 & 0.9 & 0.48 & 1.23 & 15.70 & $\mathrm{p}<0.001$ \\
\hline Control & 30 & 1.77 & 0.57 & 30 & 0.66 & 0.48 & 1.1 & 19.75 & $\mathrm{p}<0.001$ \\
\hline
\end{tabular}

The mean scores of Twakjadyata before treatment were 2.53 and 1.77 for the study and control groups respectively. After treatment, it decreased to 0.90 and 0.66 for study and control group respectively. The mean difference noticed was 1.23 and 1.1 respectively. The paired ' $t$ ' value 15.70and 19.75respectively shows that this mean difference is significant at $p<0.001$ for both study and control group.

Table 37: Effectiveness on Eosinophil count between two groups

\begin{tabular}{|c|c|c|c|c|c|c|c|c|c|}
\hline \multirow{2}{*}{ Group } & \multicolumn{3}{|c|}{ BT } & \multicolumn{3}{|c|}{$\mathbf{A T}$} & \multirow{2}{*}{$\begin{array}{c}\text { Mean } \\
\text { Diff. }\end{array}$} & \multirow{2}{*}{ Paired't' } & \multirow{2}{*}{$\mathbf{P}$} \\
\hline & $\mathbf{N}$ & Mean & SD & $\mathbf{N}$ & Mean & SD & & & \\
\hline Study & 30 & 15.4 & 3.52 & 30 & 9.7 & 2.69 & 5.7 & -18.10 & $\mathrm{p}<0.001$ \\
\hline Control & 30 & 15.73 & 3.04 & 30 & 9.63 & 2.30 & 6.1 & 23.09 & $\mathrm{p}<0.001$ \\
\hline
\end{tabular}

The mean scores of Eosinophil count before treatment were 9.7and 15.73 for the study and control groups respectively. After treatment, it decreased to 15.4 and 9.63 for study and control group respectively. The mean difference noticed was 5.7and 6.1 respectively. The paired ' $t$ ' value -18.10 and 23.09 respectively shows that this mean difference is significant at $p<0.001$ for both study and control group.

\section{Discussion}

The retrospective analysis of the resource materials reveals that specific references about Latent Poison (Dooshivisha) are not available in Vedic and allied literature. In classical Ayurvedic literatures we won't get much detailed explanation about the concept of Latent Poison (Dooshivisha). Only in Sushruta Samhita Kalpasthana $2^{\text {nd }}$ chapter we get explanation about Latent Poison (Dooshivisha), though they are not sufficient to understand the concepts of Latent 
Poison (Dooshivisha) that is regarding etiology, pathogenesis, clinical outcome and the management of Latent Poison (Dooshivisha). ${ }^{[8]}$

Any poison can get converted to Latent Poison (Dooshivisha) when they lose their characteristic ten qualities and thereby potency. Thus it is clear that the concept of Latent Poison (Dooshivisha) is based on the potency of the poison. No author has classified the Latent Poison (Dooshivisha) as a separate type of poison. In Dalhana's commentary it is mentioned as 'manda shaktikam Dooshivishatam prapnoti', (weak poisons become transformed to Dooshivisha), which very well explains about the potency of the poison.

Latent Poison (Dooshivisha) is having a very extensive range of effects on body. Symptoms of concerned Dhatu dushti is seen where the Latent Poison (Dooshivisha) is deposited, as if Latent Poison (Dooshivisha) is deposited in Rasa dhatu then Rasa dhatudushti lakshanas are seen, if in Raktadhatu, Raktadhatu dushti lakshanas can be seen and so on. To see Kushtha as a clinical entity latent Poison (Dooshivisha) should deposited in Rakta dhatu and in Twak can cause Kushtha (skin disorder) and its specific types.

While the effects of industrialization and commercialization are widely and rapidly spreading in the society, the chances of contact with toxins are increase day by day. Ayurvedic texts describes the possibilities of contacting poison from the objects used for massage, cosmetics, footwear, dress, bed, armors etc. and also prescribes specific formulations in each of such situations. These drugs may be tried in the common allergies caused by such products in the present era.

While observing the symptoms of this contact dermatitis, whatever may be the mode of poisoning, all causes localized symptoms pertaining to the skin itself, which may result immediate or delayed (latent) effect, it depends on the potency of the toxin. Even today we can notice the same effects when we use objects which contain chemicals as toxins.

The chemical substances which are consumed therapeutically for longer period of any systemic disorder, they show adverse reactions on skin as a target organ and also which are coming in contact with the skin as a toxin will definitely shows reactions to the skin itself.

Our classics have not mentioned about the particular nature of skin disorder caused by Dooshivisha (Latent poison). It is highly impossible to correlate the exact skin disorder as mentioned in Ayurveda with Contact dermatitis. As Charaka says, skin disorders always caused by three humors (Doshas) and they are innumerable, based on the symptoms pertaining to particular humor (Dosha) we should plan the treatment.

The present study, Pancharishta churna was selected by taking in consideration that, this drug is having vishghna, vranaropan, shothhar, vedanashamak and yakrututejak properties. Because of these properties this drug acts against dooshivishajanya contact dermatitis. Also many modern research papers have also supported that, contains of this drug are safest. By considering all above points, this drug was selected for the treatment of Contact dermatitis.

The literature review consists of baseline data of the disease from Ayurveda and Modern texts, journals, earlier research works and other online searches. 
A Controlled Open prospective trial was carried out on 60 patients of CONTACT DERMATITIS who were attended OPD \& having history of DOOSHIVISH POISONING.30 patients were treated by MEDICATION in Group A and 30 patients were treated by MEDICATION+ RAKTAMOKSHANA in Group B at S.T.R.H.Pune.

Both subjective and objective parameters were considered for the assessment of response to the treatment. The observations made before and after treatment were considered for analysis. Statistical analysis was carried out using paired ‘ $t$ ' test. ${ }^{[9]}$

The demographic data showed that majority of the patients were aged between 31-50 yrs of about $51.67 \%$. This is may be due to chronic nature of the disease and also indicates the incidence of Dooshivisha is more in this age group. Male predominance was seen about $66.67 \%$ in study group and $70 \%$ in control group indicates that males are more prone to suffer from Dooshivisha poisoning because of more exposure to the secondary aggravating factors. Religion wise distribution shows that most of the patients were Hindus; this may be because of small sample size. The Economic status wise distribution shows that majority of patients from poor or lower middle class. This may be because of poor living standards \& poor hygienic conditions.

Based on the personal history $-63.33 \%$ were non- vegetarians in the study group and $70 \%$ were in the control group, suggests that fish, egg, curd and meat should be considered as Dooshivisha factors, as they are mentioned in the classics as ahita and their daily usage is contraindicated. Such food will aggravate kapha, pittadoshas and responsible factors for srotorodha.

$56.67 \%$ were constipated in study group and $70 \%$ were constipated in control group; this suggests that improper expulsion of mala is also one of the aggravating factor of Dooshivisha due to accumulation of endotoxins in the body.

$70 \%$ had mental stress in the study group and $63.33 \%$ in the control group which acts as an aggravating factor. $48.33 \%$ of the patients having vatakaphaprakruti which indicates the doshic predominance of vatakapha in Dooshivishjanya contact dermatitis.

Whenever patients were asked about dietary aggravating factors, it was found that in most cases in Vegetarians patient could not link any particular dietary factor for aggravation of the disease whereas in Non-vegetarians $38.33 \%$ of patients linked that symptoms aggravates with intake of non-veg. food.

$43.33 \%$ were in occupations requiring heavy work in the study group and 43.33 in the control group. Painters, Mechanics, Farmers, Factory workers, Building workers were consider as heavy worker. They are continuous contact with cement or paint or metals or chemicals according to their occupation. Those occupational allergens were increases the prevalence of Contact dermatitis.

Alcohol consumption was found in $26.67 \%$ patients in the study group and $23.33 \%$ in the control group. This may be an added toxic effect for the accumulation in the body. 


\section{Effect of therapy on chief complaints of contact dermatitis in both groups}

- Effect on Kandu: Kandu mean score was reduced by 2.33 to 0.60 showing $74.25 \%$ relief in Study group and from 2.3 to 0.43 showing $81.30 \%$ relief in Control group. Both are individually significant $\mathrm{P}<0.001$. Therefore Raktamokshana is more effective on kandu in contact dermatitis.

- Effect on Vedana: Vedana mean score was reduced by 1.88 to 0.43 showing $77.13 \%$ relief in Study group and from 1.43 to 0.3 showing $79.02 \%$ in Control group and both are statistically significant $(\mathrm{P}<0.001)$. Therefore Raktamokashan is more effective on vedana in contact dermatitis.

- Effect on Daha: Daha mean score was reduced by 1.7 to 0.53 showing $68.82353 \%$ relief in Study group and from 1.27 to 0.27 showing $78.74016 \%$ in Control group and both are statistically significant $(\mathrm{P}<0.001)$. Therefore Raktamokashan is more effective on Daha in contact dermatitis.

- Effect on Srava: Srava mean score was reduced from 1.8 to 0.57 showing $68.33 \%$ relief in Study group and from 1.53 to 0.4 showing $73.86 \%$ relief in Control group, both are statistically highly significant $\mathrm{p}<0.001$. Pancharishta churna is having Pippali \& Maricha as ingredients which are kledahara in nature which eliminates the utkleshitadosha produce by viruddhara. Haridra is varshodhak and vishghna. This may be the reason that Study drug + Raktamokshan have been given better result.

- Effect on Pidaka: Pidaka mean score was reduced from 1.8 to 0.57 showing $68.33 \%$ relief in Study group and from 1.43 to 0.33 showing $76.92 \%$ relief in Control group, both are statistically highly significant $\mathrm{p}<0.001$. It shows that Pancharishta churna is highly effective in Pidaka when it is used along with Raktamokshana.

- Effect on Twakvaivarnya: Twakvaivarnya mean score was reduced from 2.1 to 0.8 showing $61.90 \%$ relief in Study group and from 1.83 to 0.63 showing $65.57 \%$ relief in Control group, both are statistically highly significant $\mathrm{p}<0.001$. It shows that Pancharishta churna is highly effective in Twakvaivarnya when it is used along with Raktamokshana.

- Effect on Twakjadyata: Twakjadyata mean score was reduced from 2.53 to 0.90 showing $64.42 \%$ relief in Study group and from 1.77 to 0.66 showing $62.71 \%$ relief in Control group, both are statistically highly significant $\mathrm{p}<0.001$. It shows that Pancharishta churna is highly effective in Twakjadyata.

Considering the SCORAD score assessment, both groups showed significant improvements.

1) People in the age group 31-50 years were more prone to Contact dermatitis.

2) The present study shows that Contact dermatitis is predominant in males because of more exposure to the secondary aggravating factors.

3) It is concluded that, people belonging to middle and lower economical strata of society were more prone to Contact dermatitis. This may be because of poor living standards \& poor hygienic conditions.

4) Mental stress plays an important role in the samprapti of Dooshivishjanya Contact dermatitis.

5) People having vatakapha prakruti were more prone to Contact dermatitis.

6) Improper expulsion of mala is also one of the aggravating factors of Dooshivisha. 
7) In Non-vegetarians $55 \%$ of patients linked that symptoms of contact dermatitis aggravates with intake of non-veg. food. This suggests that consumption of Non-veg. may act as a triggering factor for Dooshivishjanya Contact dermatitis.

8) Oral medication of pancharishta churna in Dooshivishjanya Contact dermatitis was found to be statistically highly significant even if it is used singly.

9) Effect of Pancharishta churna on symptoms like Pidaka, Srava, Twakvaivarnya \& Twakjadyata is highly significant when it is used along with Raktamokshana. Whereas Raktamokshan is highly significant in daha, vedana and kandu.

\section{Limitations}

1) Sample size was very small.

2) Period of the study was of short duration.

3) Follow up was done only for twenty eight days.

\section{Recommendations}

1) The study in larger samples and longer follow up at least for one year is required.

2) Concept of Dooshivisha should be understand properly and attempt to be made in the treatment of various disorders.

3) Cumulative toxicity study of particular substance is much valuable.

\section{Conclusion}

From this particular study some conclusions are being drawn on the basis of observations made, results achieved and by thorough discussion.

- Raktamokshan in Dooshivishjanya contact dermatitis was found to be statistically highly significant for Kandu, vedana and daha even if it is used singly.

- Effect of Pancharishta churna on symptoms like Pidaka, Srava, Twakvaivarnya and Twakjadyata is highly significant when it is used along with Raktamokshan.

- Raktamokshan helps in detoxifying the blood by its Shodhana Karma.

- Though Pancharishta churna is effective in Dooshivishjanya contact dermatitis its efficacy is significantly enhanced by Raktamokshan.

- This study shows that Pancharishta churna highly effective in contact dermatitis on symptoms like pidaka, srava, twakvaivarnya and twakjadyata. Raktamokshana is highly effective in contact dermatitis on symptoms like Kandu, daha, vedana.

\section{References}

[1] Dr.Anant Ram Sharma edited with 'susrutavimarsini' Hindi commentary. (1 ${ }^{\text {st }}$ Ed.). Susruta samhita,maharshi susruta. Sutra-stan; vedottpatti-adhyaya: Chapter 1.verse no.14.Varanasi : Chukhambha prakashan, 2010 ; page no.9.

[2] Dr.Y.G.Joshi, Charak Samhita of maharshi charak, Chakrapanidatta, commentator Charaka samhita, 5th ed. Varanasi: Chaukambha Sanskrit sansthana; 2001.Chikitsa sthana, visha-Chikitsa Adhyaya 23/1; page no.503. 
[3] Dr.Y.G.Joshi, Charak Samhita of maharshi charak, Chakrapanidatta, commentator Charaka samhita, 5th ed. Varanasi: Chaukambha Sanskrit sansthana; 2001.Chikitsa sthana, visha Chikitsa Adhyaya 23/2; page no.503.

[4] Dr.Anant Ram Sharma edited with 'susrutavimarsini' Hindi commentary. (1 ${ }^{\text {st }}$ Ed.). Susruta samhita,maharshi susruta. kalpa-stan; sthawarvishavidnyaniya-adhyaya: Chapter 2.verse no.33.Varanasi : Chukhambha prakashan, 2010 ; page no.524.

[5] Skin diseases \& sexually Trasmitted infection,dr.uday khopkar, $\left(5^{\text {th }}\right.$ edi),balani publication,eczema,chapter no.9,page no.99.

[6] dr.vidyanath \& nishteshwar, Sahatrayogam, ( $2^{\text {nd }}$ edition), chapter no.4,choorna prakarna,choorna no-,9,Varanasi : Chukhambha prakashan, 2008 ; page no.169

[7] Dr.Anant Ram Sharma edited with 'susrutavimarsini' Hindi commentary. (1 ${ }^{\text {st }}$ Ed.). Susruta samhita,maharshi susruta. kalpa-stan; sthawarvishavidnyaniya -adhyaya: Chapter 2.verse no.3032.Varanasi : Chukhambha prakashan, 2010 ; page no. 523.

[8] Dr.Anant Ram Sharma edited with 'susrutavimarsini' Hindi commentary. ( $1^{\text {st }}$ Ed.). Susruta samhita,maharshi susruta. kalpa-stan; sthawarvishavidnyaniya-adhyaya: Chapter 2.verse no.33.Varanasi : Chukhambha prakashan, 2010 ; page no.524.

[9] Dr.Dnyaneshwar jadhav, "Manual of research methodology and medical statistic"( $1{ }^{\text {st }}$ edition) Chapter 10 ,parametric tests ; Varanasi : Chukhambha prakashan, 2017 ; page no.240.

\footnotetext{
*Corresponding author.

E-mail address: priyashinde2000@gmail.com
} 